V.K. Kapahi and C.R. Subrahmanya

Tata Institute of Fundamental Research, P.B. No. 1234 , Bangalore 560012, India.

Possible evidence that the linear sizes of extragalactic radio sources were smaller at earlier epochs was first provided by the angular size redshift $(\Theta-z)$ relation for double radio quasars (Legg 1970, Miley 1971, Wardle and Miley 1974). But because of the strong correlation between redshift $(\mathrm{z})$ and radio luminosity $(\mathrm{P})$ in flux limited radio samples, it is hard to decide if the observed decrease in sizes with $\mathrm{z}$ is caused by an epoch dependence of linear sizes $(l)$ or by an inverse correlation between $P$ and $\ell$. Several authors (eg. Stannard and Neal 1977, Wardle and Potash 1977, Hooley et al. 1978, Wills 1979, Masson 1980) have attempted to separate the two effects by comparing the properties of quasars from the $3 \mathrm{CR}$ survey with those from the $4 \mathrm{C}$ and Parkes samples. Although most of these studies appear to marginally favour a $\mathrm{P}-l$ correlation, none of them can rule out even a fairly strong evolution in $\ell$ with $z$. Apart from the small numbers involved, the difficulty is that $3 \mathrm{C}$ and $4 \mathrm{C}$ quasars do not differ a great deal in their redshifts or luminosities. A complete sample of quasars at much weaker flux levels would be quite valuable in this regard.

Evolution in $\ell$ can be investigated also by studying the angular size-flux density ( $\Theta-S$ ) relation (Swarup 1975, Kapahi 1975). Although S in this case has to be related to $z$ through models of the evolving radio luminosity function that explain the observed source counts, there is the advantage that large unbiased samples of sources covering a wide range in $\&$ can be used. Earlier studies of the $\Theta-S$ relation, based generally on the median values of angular size $\left(\Theta_{\mathrm{m}}\right)$, have indicated (Kapahi 1975, 1977; Swarup and Subrahmanya 1977; Katgert 1977) the presence of size evolution that can be simply represented by $\ell(z) \propto(1+z)^{-n}$ with $n=1$ to 2 . Recent measurements of the structures of several complete samples have not only extended the $\Theta_{m}-S$ relation to lower flux densities but have also led to improved distributions of angular sizes at all flux levels. Furthermore, there has been a significant advancement in the optical identification of sources in the 3CR sample (Gunn et al. 1981, and references therein) which has led to improved models of the evolutionary behaviour of the radio luminosity function. It is now worthwhile, therefore, to reexamine the $\theta_{-}$; relation, and in particular to determine

$$
401
$$

D. S. Heeschen and C. M. Wade (eds.), Extragalactic Radio Sources, $401-410$. Copyright $\odot 1982$ by the IAU. 
if an inverse correlation between $P$ and $l$ can obviate the need for size evolution.

\section{THE OBSERVED $\Theta-S$ RELATION}

We shall confine ourselves to samples selected at low frequencies, in the range of 178 to $408 \mathrm{MHz}$. Such samples contain few sources of the compact 'flat spectrum' variety, so that we are dealing mainly with the extended 'steep spectrum' variety of sources. A new determination of the $\Theta_{m}-S$ relation from the best available data is shown in Figure 1. A few remarks about the samples used in constructing the relation. The highest flux density point is based on the 37 sources in the 'All Sky' catalogue (Robertson 1973) with $15 \mathrm{Jy}<\mathrm{S}_{408}<68 \mathrm{Jy}$, and $\delta<10^{\circ}$; it is therefore independent of the 3CR sample with $\delta>10^{\circ}$. The $3 \mathrm{CR}$ points are derived from the ' 166 source' sample (Jenkins et al. 1977) with the addition of 7 other sources (see next section). The $4 \mathrm{C}$ point comes from a complete sample of 72 sources in the range of 4.8 to $9 \mathrm{Jy}$ at $178 \mathrm{MHz}$, structures for which have been reported by Katgert-Merkelijn et al. (1980). The points labelled 'B2' are based largely on new observations of several complete samples (Kapahi 1981b, Padrielli et al. 1981) from the Bologna catalogues, made with the Westerbork synthesis telescope at $5 \mathrm{GHz}$. B2 sources in the upper two flux ranges ( 3.5 to $10 \mathrm{Jy}$ at $408 \mathrm{MHz}$ ) have a considerable overlap with the $4 \mathrm{C}$ and $3 \mathrm{CR}$ sources. The observed distribu-

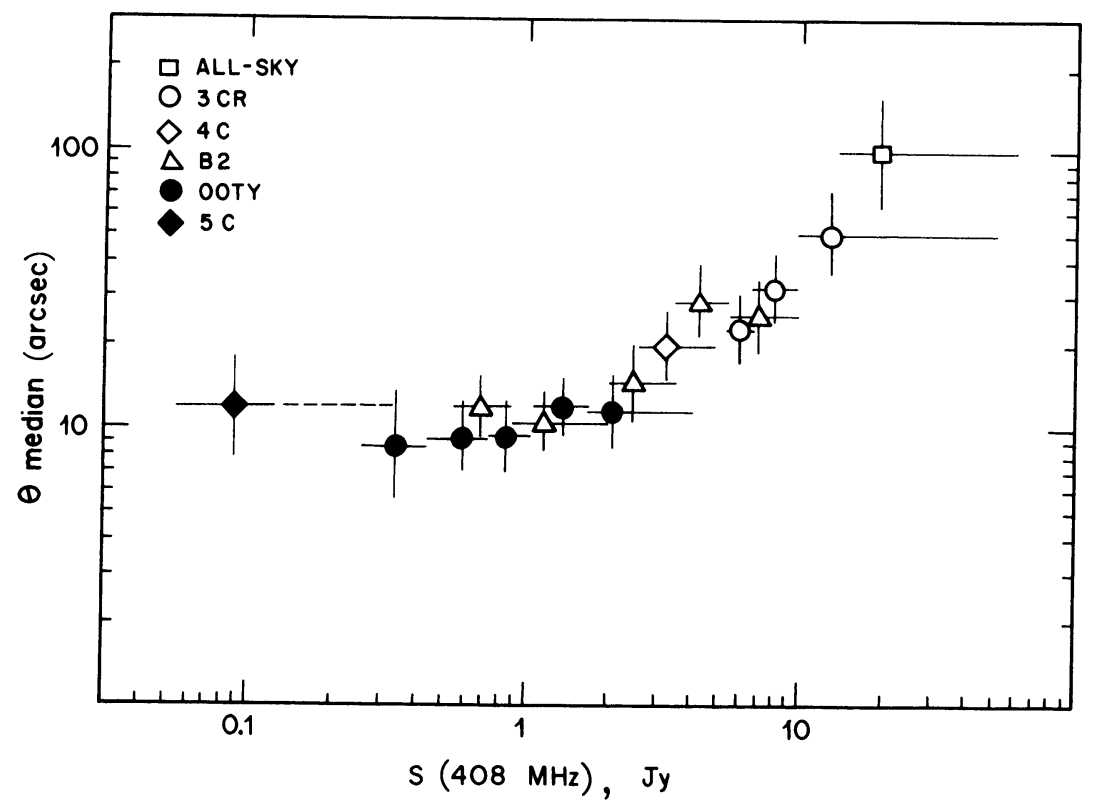

Fig. 1. The observed relation between median values of $S$ and $\theta$. The estimated errors in $\Theta_{m}$ are indicated by vertical lines and the ranges in flux density by horizontal. lines. 
tions of $\Theta$ in the other three flux ranges between 0.55 and $3.5 \mathrm{Jy}$ are shown in Figure 3. The 5 loty points, in the range of 0.3 to $5 \mathrm{Jy}$ at $327 \mathrm{MHz}$, have now been rederived from all the published occultation results to date (Joshi and Singal 1980, and references therein). The lowest flux density point is from recent VLA measurements (Downes et al. 1981) at $1465 \mathrm{MHz}$, of a complete sample of 24 sources in the $5 \mathrm{c} 6$ and 507 regions with $\mathrm{S}_{408}>0.055 \mathrm{Jy}$. The $\Theta$ distribution for this sample is also shown in Figure 3. The flux densities of samples at 178 and $327 \mathrm{MHz}$ have been translated to $408 \mathrm{MHz}$ using a constant spectral index of $\alpha=0.75$.

It is clear from Fig. 1 that the value of $\theta_{m}$ decreases fairly rapidly down to about $1-2 \mathrm{Jy}$ at $408 \mathrm{MHz}$ and remains nearly constant at lower flux levels. It should also be noted that there is good agreement between the Ooty and Bologna points that cover a similar range in $S$ but are based on almost entirely different sources. A comparison of the $\Theta$-distributions in the two sets of data indicates that though a few large sources $\left(\Theta \gtrsim 60^{\prime \prime a r c}\right)$ could have been missed in the ooty occultation surveys, their number cannot be large enough to affect the median values of $\Theta$ significantly.

\section{COMPARISON WITH PREDICTIONS OF EVOLUTIOIJARY MODELS}

In order to understand the observed $\theta$ distributions we have calculated the distributions expected at lower flux levels from the known properties of sources in the $3 \mathrm{CR}$ sample by the method that has been described in detail by Downes et al. (1981). The method uses the observed values of $\mathrm{S}, \alpha, \mathrm{z}$ and $\Theta$ for each source in the $3 \mathrm{CR}$ parent sample to estimate its contribution to the number and $\theta$-distribution in any specified range of flux density. Apart from taking into consideration the evolution in number density of radio sources according to the successful evolutionary models, the method has the advantage that any correlation between $\mathrm{P}$ and $\ell$ in the $3 \mathrm{CR}$ sample is automatically taken into account.

For the parent sample we have used both, the complete '166 source' sample with $\mathrm{S}_{178} \geq 10 \mathrm{Jy}$ and also a subsample with $\mathrm{S}_{178} \geq 15 \mathrm{Jy}$. As the 3CR sample is known to be incomplete (eg. Véron 1977), however, particularly with regard to sources of large $\Theta$, we have added the following 7 sources to the sample, for which fairly complete information is now available; DA240 ( $\left.S_{178}=19.4 \mathrm{Jy}, \Theta=2040 " \operatorname{arc}\right), 4 \mathrm{C} 73.08$ ( $\left.15.0 \mathrm{Jy}, 1100^{\prime \prime}\right)$, 4C64.19 (10.7 Jy, 120"), 4C35.40 (15.2 Jy, 840"), NGC6251 ( $10.0 \mathrm{Jy}$, 4320"), 4C11.71 (11.1 Jy, 720"), 3C296 (13.0 Jy, 208"). Furthermore, we take the total flux densities of 30236 and 36326 to be 19.8 and 16.7 Jy respectively, values that are considerably larger than those listed in Jenkins et al. (1977).

Redshifts are now known for about $75 \%$ of the sources in the 3CR sample. For most of the remaining these have been estimated from the magnitudes of the identified galaxies. For the unidentified sources and 
those identified with objects of $m>22$, we assume $z=1$. The uncertainty in these redshifts makes little difference to the results. The computations were done numerically for the 3 successful evolution models of Wall et al. (1980; WPL) (labelled as models ' $4 \mathrm{a}^{\prime}$ ', ' $4 \mathrm{~b}$ ' and ' 5 ' by WPL), and for various amounts of linear size evolution assumed to be represented by $(1+z)^{-n}$. Values of $H_{O}=50 \mathrm{~km} \mathrm{sec}^{-1} \mathrm{Mpc}^{-1}$ and $\mathrm{q}_{\mathrm{O}}=0.5$ were used; $\mathrm{q}_{\mathrm{O}}=0$ was also used for models ' $4 \mathrm{~b}$ ' and ' 5 ' (model ' $4 \mathrm{a}$ ' does not give a satisfactory fit to the source counts for $q_{0}=0$; WPL and C.R. Subrahmanya, in preparation). The results of using the entire 3CR sample and the subsample with $\mathrm{S}>15 \mathrm{Jy}$ are quite similar but the errors in the predicted numbers should be smaller for the larger sample.

The predicted $\Theta_{m}-S$ relations (from the $S \geq 15$ Jy sample, $q_{0}=0.5$ ) for the three evolutionary models without size evolution $(n=0)$ are shown in Figure 2. It can be seen that these predict significantly larger values of $\Theta_{m}$ than observed. But with size evolution included, satisfactory fits to the data can be obtained in all the models for the values of $\mathrm{n}$ indicated in Fig. 2. It is also interesting to note that rather strong size evolution $(\mathrm{n} \sim 3.5)$ is needed for model ' 5 ' of WPL. This is because the average redshift of sources in this model at all flux densities is considerably smaller than in the other models. It has been shown recently by Swarup et al. (1981) that model 5 is inconsistent with optical identification data because it predicts a much higher rate

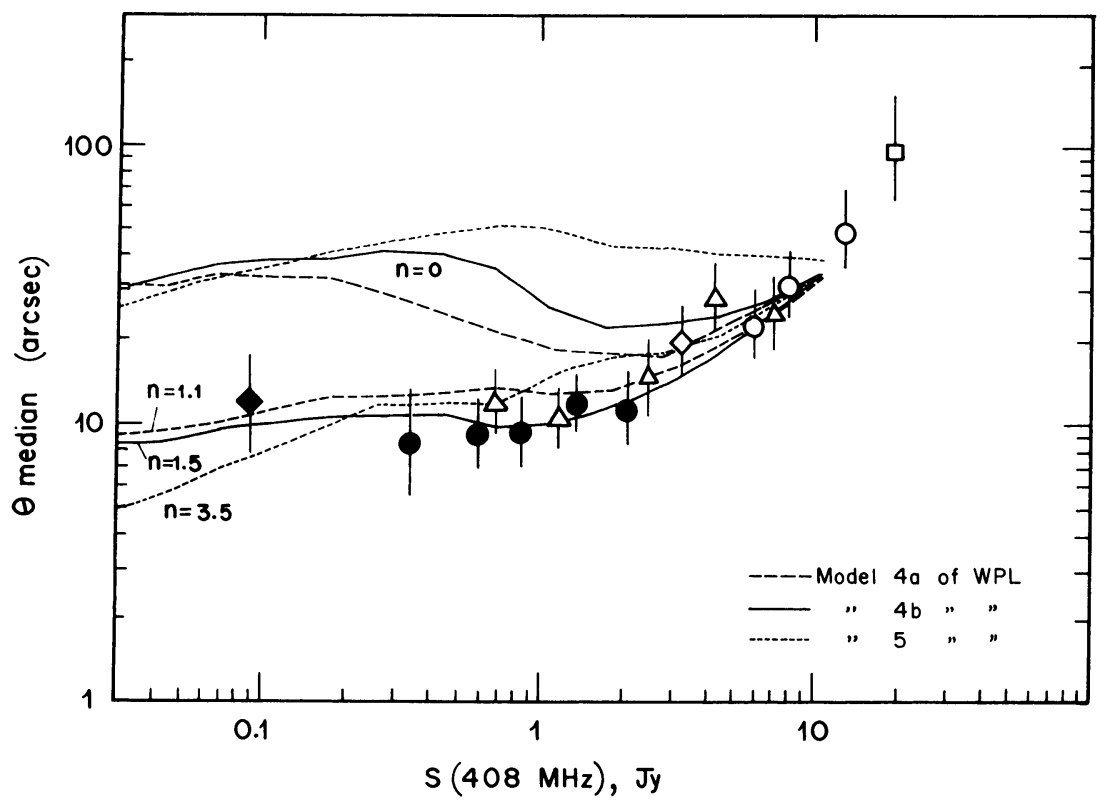

Fig. 2. The $\Theta_{m}-S$ relations predicted from the $3 \mathrm{CR}$ sample ( $\mathrm{S}>15 \mathrm{Jy}$, $\left.q_{0}=0.5\right)$ for three evolutionary models of WPL, without size evolution (upper curves) and with size evolution denoted by the values of index $n$ as indicated (lower curves). 
of percentage identification in the entire range of flux densities than is observed. The strong size evolution required in this model appears to be inconsistent with the observed $\Theta-z$ relation for quasars and thus provides additional evidence against the model.

The observed and predicted distributions of $\theta$ in 4 complete samples ( $5 \mathrm{C}$ and $\mathrm{B} 2$ ) that have no overlap with the $3 \mathrm{CR}$ sources are shown in Fig. 3 for model ' $4 b^{\prime}$ ', with and without size evolution. While the agreement appears to be quite good throughout the observed range of $\Theta$ at different flux levels for the case with size evolution, too few sources with small angular sizes ( $\theta \leqslant 6$ "arc) and too many with large sizes $\left(\Theta z\right.$ 40"arc) are predicted in the absence of size evolution. A $x^{2}$ test of the observed and predicted distribution (from the entire 3CR sample) indicates (see Table 1) that these are indeed significantly different for the case of $n=0$. In performing the $x^{2}$ test the lowest $\theta$-bin in each flux range was chosen to include the upperlimits to $\Theta$ for the unresolved sources. At higher $\Theta$ values an interval of 0.2 in log $\Theta$ was used and neighbouring bins marged where necessary so as to have at least 5 sources for the comparison. There are thus 26 bins in $\log \Theta$, but since the total predicted number of sources in each flux range has been normalized to agree with the observed number (the differences are however within the expected standard errors), there are 22 degrees of freedom. It is also apparent from Table 1 that size evolution is needed even in the case of a $q_{0}=0$ Universe.

Table 1. $x^{2}$ analysis of $\Theta$ distributions (degrees of freedom $=22$ )

\begin{tabular}{ccccc}
\hline$q_{0}$ & model & $n$ for $\min \cdot x^{2}$ & $x^{2} \min$ & $x^{2}(n=0)$ \\
\hline 0.5 & $4 a$ & 1.1 & 19 & 38 \\
& $4 b$ & 1.4 & 12 & 49 \\
& 5 & 3.6 & 19 & 122 \\
0.0 & $4 b$ & 1.1 & 14 & 33 \\
& 5 & 2.5 & 16 & 63 \\
\hline
\end{tabular}

The results shown in Figs. 2 and 3 do not agree with those of Downes et al. who concluded on the basis of predictions from the $\mathrm{S} \geqslant 15 \mathrm{Jy}$ 3CR sample that no single value of $n$ provides even a tolerable fit to the observed $\Theta_{m}$ values over the entire flux density range in any of the WPL models, and that for $n>1$ insignificant numbers of sources with large sizes $\left(\Theta \gtrsim 25\right.$ "arc) are predicted. The $\Theta_{m}$ values predicted by us from the same $3 \mathrm{CR}$ sample with identical assumptions regarding the redshifts of those sources that do not have measured values and for the same values of $n$ used by them $(n=0$ and $n=1)$ appear infact to be considerably different throughout the flux density range for all three WPL models considered. The reason for the discrepancy is unclear. We are reasonably confident, however, that a computational oversight on our part is unlikely to be the cause. 


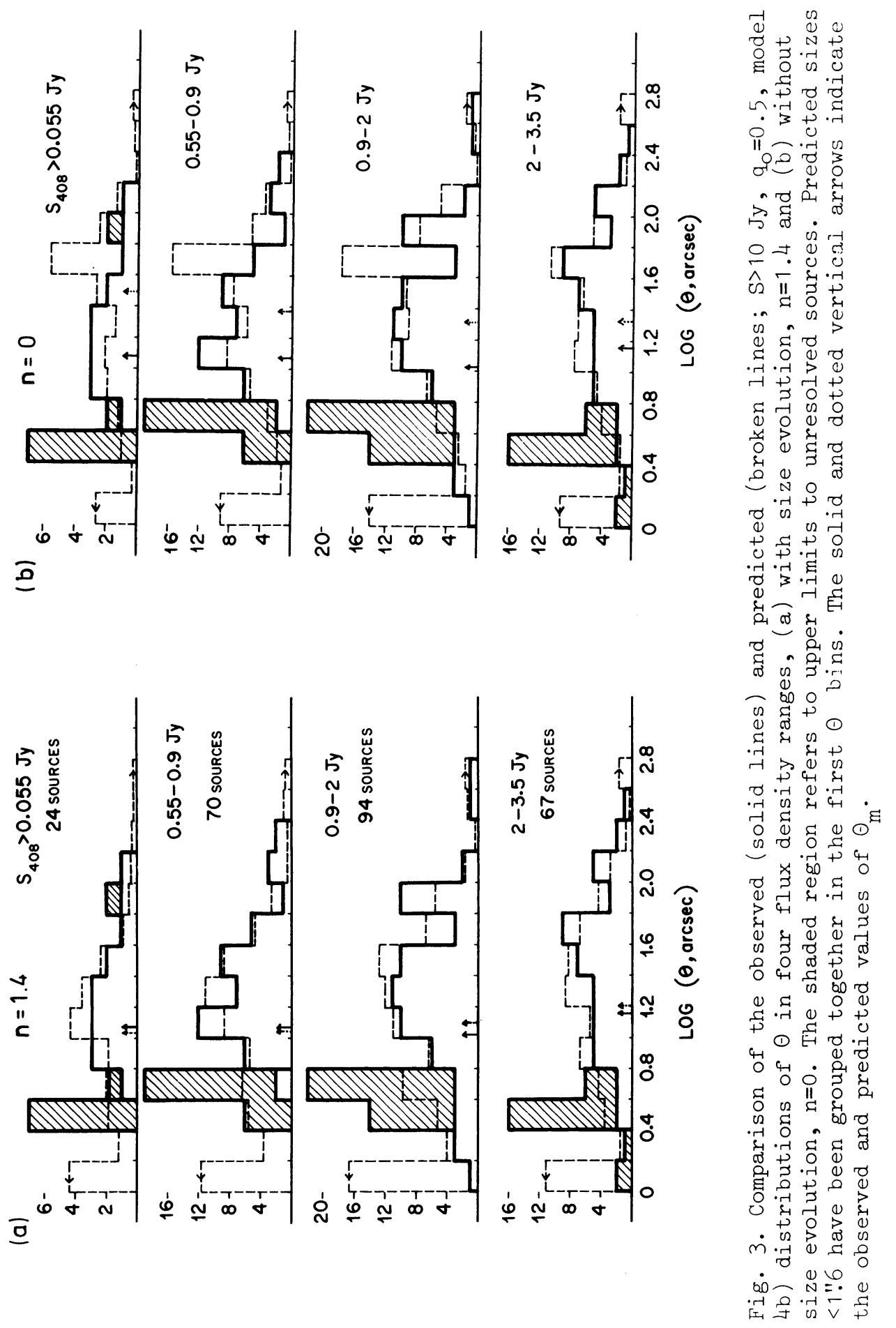


It has been suggested by Downes et al. that the fairly large number of unresolved sources ( $\theta \leqslant 3$ "arc) in the $5 \mathrm{C}$ samples could be similar to the compact steep spectrum sources that form a significant fraction of the strong source population at high frequencies (e.g. Kapahi 1981a, Peacock and Wall 1981) but are underrepresented in the 3CR sample because of low frequency turnovers in their radio spectra. Such sources are unlikely, however, to obviate the need for size evolution because such evolution appears to be needed to explain the observed $\Theta$-distributions at large angular sizes as well. Our analysis also shows that over $90 \%$ of the counts with $\Theta<3$ "arc in the $5 \mathrm{C}$ sample are contributed by galaxies in the 3CR sample that have $z<0.5$ and angular sizes of upto about 20"arc. Most of the compact steep-spectrum sources found in strong source surveys at frequencies of 2.7 or $5 \mathrm{GHz}$ on the other hand are either quasars or have $m>20$. It is therefore unlikely that such sources make a substantial contribution to the counts of unresolved sources at the 5C flux levels.

\section{CONCLUSIONS}

The available angular size data down to $55 \mathrm{mJy}$ at $408 \mathrm{MHz}$ appear to require evolution in linear sizes of the powerful radio sources. The likely value of $n$ if the evolution is expressed as $(1+z)^{-n}$, appears to lie in the range of about 1 to 1.5. Size evolution is needed in addition to any $\mathrm{P}-l$ correlation implicit in the $3 \mathrm{CR}$ data. Such a correlation alone is not sufficient because the best available evolutionary models of the radio luminosity function imply that the median luminosity of radio sources remains almost constant in the flux density range of about 10 to $1 \mathrm{Jy}$ at $408 \mathrm{MHz}$ where $\Theta_{\mathrm{m}}$ decreases by a factor of $\sim 3$. The median redshift of sources in this range of flux density increases typically from about 0.35 to $\gtrsim 1$, but since for a constant linear size, $\Theta$ decreases rather slowly with increasing $z$ in world models with $q_{0}>0$, a decrease in $\ell$ with increasing $z$ is required to fit the observations.

It would be useful to measure the structures of unresolved sources in the present samples with much higher angular resolution in order to improve the distributions at small angular sizes and to determine the nature of the compact steep-spectrum sources.

\section{REFERENCES}

Downes, A.J.B., Longair, M.S. and Perryman, M.A.C. 1981, Mon. Not. R. astr. Soc., in press.

Gunn, J.E., Hoessel, J.G., Westphal, J.A., Perryman, M.A.C. and Longair, M.S. 1981, Mon. Not. R. astr. Soc., 194, 111.

Hooley, T.A., Longair, M.S. and Riley, J.M. 1978, Mon. Not. R. astr. Soc., 182, 127.

Jenkins, C.J., Pooley, G.G. and Riley, J.M. 1977, Mem. R. astr. Soc., $84,61$.

Joshi, M.N. and Singal, A.K. 1980, Mem. Astron. Soc. Inaia, 1, 49. 
Kapahi, V.K. 1975, Mon. Not. R. astr. Soc., 172, 513.

Kapahi, V.K. 1977, in IAU Symposium 74 "Radio Astronomy and Cosmology", ed. D. Jauncey, p. 119.

Kapahi, V.K. 1981a, Astron. Astrophys. Suppl., 43, 381.

Kapahi, V.K. 1981b, in preparation.

Katgert, P. 1977, Ph. D. Thesis, University of Leiden.

Katgert-Merkelijn, J., Lari, C. and Padrielli, L. 1980, Astron. Astrophys. Suppl., 40, 91.

Legg, T.H. 1970, Nature, 226, 65.

Masson, C.R. 1980, Astrophys. J., 242, 8.

Miley, G.K. 1971, Mon. Not. R. astr. Soc., 152, 477.

Padrielli, L., Kapahi, V.K. and Katgert-Merkelijn, J.K. 1981, Astron. Astrophys. Suppl., in press.

Peacock, J.A. and Wall, J.V. 1981, Mon. Not. R. astr. Soc., in press. Robertson, J.G. 1973, Austr. J. Phys., 26, 403.

Stannard, D. and Neal, D.S. 1977, Mon. Not. R. astr. Soc., 179, 719.

Swarup, G. 1975, Mon. Not. R. astr. Soc., 172, 501.

Swarup, G. and Subrahmanya, C.R. 1977, in IAU Symposium 74 "Radio Astronomy and Cosmology", ed. D. Jauncey, p. 125.

Swarup, G., Subrahmanya, C.R. and Venkatakrishna, K.L. 1981, Astron. Astrophys., submitted.

Véron, P. 1977, Astron. Astrophys. Suppl., 30, 131.

Wall, J.V., Pearson, T.J. and Longair, M.S. 1980, Mon. Not. R. astr. Soc., 193, 683.

Wardle, J.F.C. and Miley, G.K. 1974, Astron. Astrophys., 30, 305.

Wardle, J.F.C. and Potash, R. 1977, Ann. NY Acad. Sci., 302, 605.

Wills, D. 1979, Astrophys. J. Suppl., 39, 291. 
DISCUSSION, of previous two papers, by Downes, and by Kapahi and Subrahmanya

SWARUP: How do you justify the use of parent sample at $2.7 \mathrm{GHz}$, for which the emitted frequency will be $\sim 5 \mathrm{GHz}$ at $\mathrm{z}=1$, for comparing with the $\theta$ - S data at $408 \mathrm{MHz}$ for the 56 sources?

DOWNES: The method of estimation of angular sizes gives a local density to each source in the parent sample proportional to $1 / \mathrm{V}_{\max }$, the maximum volume in which the source would have been included in the sample. This means that high weight is given to low-luminosity, low-redshift sources, for which the emitted frequency $(1+z) 2.7 \mathrm{GHz}$ would be close to $2.7 \mathrm{GHz}$. Contributions from high-redshift sources are small both because $V_{\max }$ is large and because it is hard to move them far enough away to reach the required low-flux densities without involving unreasonably large redshifts. The $5 \mathrm{C}$ sources, however, are likely to lie at $z>0.6$ so would have an emitted frequency $\sim 1 \mathrm{GHz}$. $2.7 \mathrm{GHz}$ is the closest frequency to this for which almost complete $\theta$ and $z$ data exist.

REES: Presumably the $\theta(z)$ relation is, to a large extent, probing the $z$-dependence of the intergalactic environment of extended radio lobes. But this environment evolves in a complex way: between clusters, the gas was probably denser in the past; within clusters, the density may be higher now (and indeed may be higher than the intergalactic density was anywhere at $z \sim 2$ ). For this reason, and others, one would not expect any simple power-law to be a good fit. Obviously these data will eventually tell us a great deal, when we have AXAF-type maps showing the properties of intergalactic gas out to large $z$. But how much do you really think these studies can now tell us about the physics of the sources?

DOWNES: It is not clear whether one would expect sources at high $z$ to have different linear sizes. Comparisons of linear sizes outside and inside clusters (i.e., high-density environments) indicate no significant differences in size. On the other hand, high inversion Compton losses may extinguish high-z sources when they are smaller. Any indication of whether high-z sources are indeed smaller would therefore add to our understanding of the source population.

WILLS: In future studies, do you consider it more profitable to restrict the observed samples to one optical class, with measured redshifts, or to continue using much larger samples that are a mixture of all optical classes?

DOWNES: Measurements of redshifts of sources in the present samples, particularly at the lowest flux densities, would place considerable contraint on radio luminosity functions, and would seem the most profitable course of action to resolve the situation. 
BALDWIN: Both speakers recognize the problem of attaining completeness for radio sources of the largest angular size. In comparing data with the models is some attempt made to use some limiting rest-frame surface brightness? If not, how much does it matter?

DOWNES: (1) No. (2) It probably doesn't matter a great dea1--at 80" resolution (in the $5 \mathrm{C}$ surveys) very few of the sources are resolved so that we do not expect a significant contribution from low-brightness extended objects. Low-brightness compact objects would simply be below the sample limits.

LAING: (1) Could the different definitions of angular size for Class I and Class II sources cause any problems? (2) Many flatspectrum "compact" sources have weak, extended outer components which are difficult to detect. If these are neglected, then the angular sizes are considerably underestimated. (3) In the 3CR sample at least, the "steep-spectrum compact sources" are of two types: (a) small symmetrical doubles, and (b) steep-spectrum radio cores. These may have different evolutionary properties.

KAPAHI : (1) The proportion of Class I radio galaxies ( $P<10^{25} \mathrm{~W}$ $\mathrm{Hz}^{-1}$ ster $^{-1}$ ) does not increase (probably decreases) with decreasing $\mathrm{S}$ in the flux range of interest because they are not subject to strong density evolution. Such sources can in any case be fairly easily recognized from the magnitudes of their optical counterparts at least down to about $1 \mathrm{Jy}$. Leaving them out from the samples (including 3CR) seems to make little difference to the conclusions. (2) Most of the sources of this type have probably been considered "unresolved" in our data. The number of flat-spectrum sources are anyway not large enough in low frequency samples to affect the results significantly.

(3) These could well be of two kinds, but little is known about their evolutionary behaviour. 